\title{
A VARIATIONAL RESULT IN A DOMAIN WITH BOUNDARY*
}

\section{YANYAN $\mathrm{LI}^{\dagger}$ AND LOUIS NIRENBERG ${ }^{\ddagger}$}

When looking for critical points of a function, which are neither minima nor maxima, the function is sometimes defined in a domain with boundary. For example, M. Struwe (see section 11 in [2]) treats functions in convex bodies. P. Majer proved a number of very nice results in [1] on Finsler manifolds with boundary. In this paper we present a rather simple result for a real function $F$ in a bounded domain. Under some conditions on the boundary values $\phi$ of $F$ we prove that $F$ has a critical point in the domain.

For simplicity we always assume that $F$ is in $C^{2}$. We start first with domain in $\mathbb{R}^{n}$, Theorem 1 , while Theorem $1^{\prime}$ considers a domain in a Hilbert space.

THEOREM 1. Let $F$ be a real $C^{2}$ function in the closure $\bar{\Omega}$ of a smooth bounded domain in $\mathbb{R}^{n}$. Assume that

$$
\phi:=\left.F\right|_{\partial \Omega}: \partial \Omega \rightarrow \mathbb{R}
$$

has only two critical values, $\max$ and $\min$. Denote by $m$ the set where $\phi$ takes its minimum. Assume:

(i) $m$ is contractible to a point in $\bar{\Omega}$.

(ii) In some $\alpha$-neighborhood on $\partial \Omega$ of $m$, $m$ is not contractible to a point.

Then $F$ has a critical point in $\Omega$.

As one would expect, the proof relies on a deformation lemma. It is a modification of the usual one, since we are working in a bounded domain; it is close to several in $[1]$.

LEMma 1. Let $\Omega$ be a smooth bounded domain in $\mathbb{R}^{n}$ and let $F$ be a real $C^{2}$ function on $\bar{\Omega}$ having no critical value in $\Omega$ in the interval $[a, b], a<b$. Assume also that

$$
\phi:=\left.F\right|_{\partial \Omega}: \partial \Omega \rightarrow \mathbb{R}
$$

has no critical value in $[a, b]$. Set

$$
\widetilde{m}=\{\text { critical points of } F \text { in } \Omega \text { where } F<a\} \cup\{\text { critical points of } \phi \text { where } \phi<a\}
$$

Consider the sets

$$
\begin{gathered}
W=\{x \in \bar{\Omega} ; F(x) \leq b\} \\
V=\{x \in \bar{\Omega} ; F(x)<a\} .
\end{gathered}
$$

Then, there is a continuous deformation of $W$ to $V$, keeping $\widetilde{m}$ pointwise fixed, that is, for some $T>0$ there is a continuous map $\eta: W \times[0, T] \rightarrow \bar{\Omega}$ such that

$$
\begin{cases}\eta(x, 0)=x & \forall x \in W \\ \eta(x, T) \in V & \forall x \in W \\ \eta(x, t)=x & \forall x \in \widetilde{m}, \quad \forall t \in[0, T] .\end{cases}
$$

\footnotetext{
* Received December 13, 1999.

†Rutgers University, Department of Mathematics, Piscataway, NJ 08854, USA (yyli@math. rutgers.edu).

$\ddagger$ Courant Institute, New York University, 251 Mercer Street, New York, NY 10012, USA.
} 
Proof. Near $\partial \Omega$ we make an orthogonal decomposition of $\nabla F$ :

$$
\nabla F=v_{1}+v_{2}
$$

where $v_{1}$ is the component of $\nabla F$ pointing normal to $\partial \Omega$. By our hypotheses, there is a neighborhood $N$ of $\partial \Omega$ and there exists $\delta>0$ such that

$$
\left|v_{2}(x)\right| \geq \delta \quad \text { for } \quad x \in(W \backslash V) \cap N .
$$

In addition, by possible reducing $\delta$, we have

$$
|\nabla F(x)| \geq \delta \text { for } \quad x \in W \backslash V .
$$

Let $\zeta$ be a real function in $C_{0}^{\infty}(\Omega)$ with $0 \leq \zeta \leq 1$ and $\zeta \equiv 1$ on $\Omega \backslash N$. Consider the flow $\eta(x, t)$ for $x \in W, t \geq 0$ defined by

$$
\frac{d \eta}{d t}=-\zeta(\eta) \nabla F(\eta)-(1-\zeta(\eta)) v_{2}(x), \quad \eta(x, 0)=x .
$$

Clearly

$$
\eta(z, t)=z \quad \text { for } \quad z \in \widetilde{m}, t \geq 0 .
$$

Note that on $\partial \Omega, d \eta / d t$ is tangential to $\partial \Omega$, so $\eta(x, t)$ always lies in $\bar{\Omega}$.

Next,

$$
\frac{d}{d t} F(\eta(t))=-\zeta(\eta)|\nabla F(\eta)|^{2}-(1-\zeta(\eta))\left|v_{2}(\eta)\right|^{2} \leq 0
$$

Thus, where $\zeta(\eta(t))=1$, we have by (4),

$$
\frac{d}{d t} F(\eta) \leq-\delta^{2}
$$

On the other hand, if $\zeta(\eta(t))<1$ and if $\eta(t)$ lies in $W \backslash V$, then (3) holds and so, again,

$$
\frac{d}{d t} F(\eta(t)) \leq-\delta^{2}
$$

It follows that if $x \in W$, then $\eta(x, t) \in W$ and, after time $t=T=(b-a) \delta^{-2}$,

$$
\eta(x, t) \text { lies in } V \text {. }
$$

Proof. [Proof of Theorem 1] We may assume that the set $M$ where $\phi$ achieves its maximum is different from $m$. Otherwise there is nothing to prove. In addition we may suppose $\phi=1$ on $M, \phi=-1$ on $m$, and that

$$
|F|<1 \text { in } \Omega \text {. }
$$

For otherwise $F$ would have a maximum or minimum in $\Omega$ and again there is nothing to prove.

Let $U$ be an $\alpha$-neighborhood of $m$ on $\partial \Omega$, in which $m$ is not contractible to a point. For possibly smaller $\alpha$, let $V_{1}$ be the set of points in $\bar{\Omega}$ whose distance to $\partial \Omega$ 
is less than $\alpha$ and such that for each $x$ in $V_{1}$ its projection $P x$ to its closest point on $\partial \Omega$, lies in $U$. Now,

$$
\text { for } 0<1+a \text { small, } V:=\{x \in \bar{\Omega} ; \quad F(x)<a\} \text { lies in } V_{1} .
$$

By condition (i), there is a continuous map

$$
R: m \times[0,1] \rightarrow \bar{\Omega}
$$

such that

$$
\begin{array}{ll}
R(x, 0)=x & \forall x \in m, \\
R(x, 1)=x_{0} \in \bar{\Omega} & \forall x \in m .
\end{array}
$$

Since we may move $R(x, t)$ a bit for $t>0$, we may also suppose that

$$
R(x, t) \in \Omega \quad \forall x \in m, \forall t \in(0,1] .
$$

In particular, we can ensure that the closed set

$$
\left\{\begin{array}{l}
K:=\{R(x, t) ; x \in m, 0 \leq t \leq 1\} \\
\text { lies in } \bar{\Omega} \backslash W \text { where } W:=\{x \in \bar{\Omega} ; F(x) \leq 1-\tau\} \text { for some } \tau>0 .
\end{array}\right.
$$

We now argue by contradiction. Suppose that $F$ has no critical point in $\Omega$. Using $V$ and $W$ as defined in (5) and (8), we apply Lemma 1 and find a deformation $\eta(\cdot, t)$ of $\bar{\Omega} \backslash W$ into $V$. Thus for $y \in K, \eta(y, T) \in V$, i.e., $\forall x \in m, \forall t \in[0,1]$, $(\eta(R(x, t)), T) \in V \subset V_{1}$. Hence

$$
D(x, t):=P(\eta(R(x, t)), T) \in U
$$

provides a deformation in $U$ of $m$ to the point $\eta\left(x_{0}, T\right)$-contradicting (ii).

REMARK 1. Condition (i) in Theorem 1 may not be dropped, as we see from the example

$$
\Omega=\{1<|x|<2\} \quad \text { in } \mathbb{R}^{2} ; \text { and } F(x)=|x| \text {. }
$$

REMARK 2. The function $F$ in Lemma 1, and hence in Theorem 1, need not be in $C^{2}(\Omega)$. It suffices that it is in $C^{1}(\bar{\Omega})$. One then uses the pseudo-derivative (see for example [2]).

We now take up an extension of Theorem 1 and of Lemma 1 to a Hilbert space $H$. Here $\Omega$ is a bounded domain (open connected set) in $H$ with smooth boundary. In $\bar{\Omega}$ we consider a real function $F$ belonging to $C^{2}(\bar{\Omega})$ and satisfying the strong condition on its Frechet derivative $F^{\prime}$;

$$
F^{\prime} \text { is uniformly continuous in some } \beta \text { - neighborhood of } \partial \Omega \text {. }
$$

With

$$
\phi:=\left.F\right|_{\partial \Omega} \rightarrow \mathbb{R}
$$


we assume that

$$
F \text { satisfies } P S(\bar{\Omega}) \text { and } \phi \text { satisfies } P S(\partial \Omega) \text {. }
$$

Here $P S$ means the Palais-Smale condition holds, i.e., for $F$, any sequence $\left\{x_{j}\right\} \in \bar{\Omega}$ such that $\left|F\left(x_{j}\right)\right|$ is bounded and $\left\|F^{\prime}\left(x_{j}\right)\right\| \rightarrow 0$, has a strongly convergent subsequence. (Similarly for $\phi$ on $\partial \Omega$ ). See for example [2].

THEOREM $1^{\prime}$. Let $\Omega$ and $F$ be as above. Assume that $\phi$ has only two different critical values, its $\max$ and $\min$; denote by $M$ and $m$ the sets where these are taken on. Assume

(i) $m$ is contractible to a point in $\bar{\Omega}$.

(ii) In some $\alpha$-neighborhood on $\partial \Omega$ of $m$, $m$ is not contractible to a point.

Then $F$ has a critical point in $\Omega$.

The proof makes use of an extension of Lemma 1 :

Lemma $1^{\prime}$. Suppose that $F$ and $\phi$ satisfy the conditions of Lemma 1. Then the conclusion of the lemma holds.

Proof. The proof is the same as that of Lemma 1. But we have to ensure that (3) and (4) hold. We see first that since $\phi$ satisfies $P S(\partial \Omega)$, for some $\delta^{\prime}>0$,

$$
\|\nabla \phi\| \geq \delta^{\prime} \quad \text { on } \quad \partial \Omega \cap(W \backslash V) .
$$

From the uniform continuity of $F^{\prime}$ we infer that there is a $\beta$-neighborhood $N$ of $\partial \Omega$ and $\exists \delta>0$ such that (3) holds:

$$
\left\|v_{2}(x)\right\| \geq \delta \quad \text { for } \quad x \in(W \backslash V) \cap N .
$$

In addition, by possibly reducing $\delta$, since $F$ satisfies $P S(\bar{\Omega})$, we find

$$
\left\|F^{\prime}(x)\right\| \geq \delta \quad \text { for } \quad x \in W \backslash V .
$$

The proof of Lemma $1^{\prime}$ then follows that of Lemma 1 and we consider Lemma $1^{\prime}$ to be proved.

Proof. [Proof of Theorem 1'] We suppose, as before, that

$$
\max \phi=1, \quad \min \phi=-1 .
$$

Then $|F|<1$ in $\Omega$, otherwise there is nothing to prove.

We follow the proof of Theorem 1. However, we have to ensure that (5) and (8) hold. These both follow from

LEMMA 2. Under the conditions of Theorem $1^{\prime}$, outside of any (relatively) open neighborhood $W$ of $M$, and $V$ of $m$, in $\bar{\Omega}$ there exists a constant $\delta^{\prime}>0$ such that

$$
-1+\delta^{\prime} \leq F(x) \leq 1-\delta^{\prime}
$$

This clearly ensures (5) and (8); hence the proof of Theorem $1^{\prime}$ is complete once Lemma 2 is proved.

Proof. [Proof of Lemma 2] Suppose $W$ and $V$ are open neighborhoods of $M$ and $m$ in $\bar{\Omega}$. Since $\phi$ satisfies $\operatorname{PS}(\partial \Omega), M$ and $m$ are compact. So, for some $\beta>0, W$ and $V$ 
contain a $\beta$-neighborhood of $M$ and $m$ respectively. We prove the second inequality in (12) by contradiction argument. Suppose that for a sequence $\left\{x_{j}\right\} \subset \bar{\Omega} \backslash W$,

$$
F\left(x_{j}\right) \rightarrow 1
$$

After passing to a subsequence (still denoted as $\left\{x_{j}\right\}$ ), there are two cases:

Case 1. For some $0<\beta^{\prime}<\beta / 2, \operatorname{dist}\left(x_{j}, \partial \Omega\right) \geq \beta^{\prime}$ for all $j$.

Case 2. $\operatorname{dist}\left(x_{j}, \partial \Omega\right) \rightarrow 0$.

In Case 1, we consider the flow

$$
\frac{d \eta}{d t}=\frac{F^{\prime}(\eta)}{\left\|F^{\prime}(\eta)\right\|}, \quad \eta(0)=x_{j} .
$$

Let $T_{j}$ be the largest number in $\left(0, \beta^{\prime} / 2\right]$ such that $\left\|F^{\prime}(\eta(t))\right\| \geq 1 / j$ for all $0 \leq t \leq T_{j}$. Pick $0 \leq t_{j} \leq T_{j}$ with

$$
\left\|F^{\prime}\left(\eta\left(t_{j}\right)\right)\right\|=\min _{0 \leq t \leq T_{j}}\left\|F^{\prime}(\eta(t))\right\|
$$

Clearly,

$$
F\left(\eta\left(t_{j}\right)\right) \rightarrow 1 \quad \text { and } \quad \operatorname{dist}\left(F\left(\eta\left(t_{j}\right)\right), M\right) \geq \beta^{\prime} / 2 .
$$

We will show that

$$
\left\|F^{\prime}\left(\eta\left(t_{j}\right)\right)\right\| \rightarrow 0 \text {. }
$$

This would lead to contradiction since from the $P S$ property for $F$ we would find a subsequence of $F\left(\eta\left(t_{j}\right)\right)$ converging to a point $y$ with $F(y)=1$ (i.e. $y \in M$ ) and $\operatorname{dist}(y, M) \geq \beta^{\prime} / 2$. Impossible.

If $T_{j}<\beta^{\prime} / 2$, then $\left\|F^{\prime}\left(\eta\left(T_{j}\right)\right)\right\|=1 / j$ and (13) follows immediately. Otherwise, $T_{j}=\beta^{\prime} / 2$, then the flow is well defined in $\left[0, \beta^{\prime} / 2\right]$, and

$$
\int_{0}^{\beta^{\prime} / 2}\left\|F^{\prime}(\eta(t))\right\| d t=F\left(\eta\left(\beta^{\prime} / 2\right)\right)-F\left(x_{j}\right) \leq 1-F\left(x_{j}\right) \rightarrow 0 .
$$

This yields (13) as well.

In case 2 , since $F^{\prime}$ is uniformly continuous near $\partial \Omega$ and $\phi$ satisfies $P S(\partial \Omega)$, there exist $\delta>0$ and $0<r<\beta / 2$ such that

$$
\left\|v_{2}(x)\right\| \geq \delta>0 \quad \forall\left\|x-x_{j}\right\| \leq r, \forall j .
$$

Consider the flow

$$
\frac{d \eta}{d t}=\frac{v_{2}(\eta)}{\left\|v_{2}(\eta)\right\|}, \quad \eta(0)=x_{j}, \quad 0 \leq t \leq r
$$

Since

$$
\frac{d}{d t} F(\eta(t))=\left\|v_{2}(\eta(t))\right\| \geq \delta \quad \forall 0 \leq t \leq r
$$

we have

$$
F(\eta(r)) \geq F\left(x_{j}\right)+\delta r=1+\delta r+o(1)
$$


Impossible for large $j$.

The right hand inequality of (12) is proved, and the other is similar.

The proofs of Lemma 2 and of Theorem $1^{\prime}$ are complete.

ExAmple. Let $\Omega$ be a ball, $\|x\|<1$, in a Hilbert space $H$. Suppose $H$ has the orthogonal decomposition

$$
H=H_{1} \oplus H_{2} \quad \text { with } \quad \operatorname{dim} H_{1}<\infty .
$$

Let $F$ be a $C^{2}$ function in $\bar{\Omega}$ satisfying $(P S)$ and $F^{\prime}$ uniformly continuous in $\bar{\Omega}$.

Suppose that

$$
\phi:=\left.F\right|_{\partial \Omega}=\left\|x_{2}\right\|^{2}-\left\|x_{1}\right\|^{2}
$$

here $x=x_{1}+x_{2}, x_{1} \in H_{1}, x_{2} \in H_{2}$. Then $F$ has a critical point in $\Omega$.

Proof. Condition (i) in Theorem $1^{\prime}$ is obvious, while condition (ii) is easily verified, since $\left.m=\left\{x_{1}, 0\right\} ;\left\|x_{1}\right\|=1\right\}$ is a finite dimensional sphere. Finally, it is easy to see that $\phi$ satisfies $P S$ on $\partial \Omega$.

QUESTION. In the preceding example suppose that both $H_{1}$ and $H_{2}$ are infinite dimensional. Does the conclusions hold?

We suspect not.

ACKNowledgment. The first author was supported by the grant NSF-DMS9706887, the second by grant NSF-DMS-9400192, and by Institut Universitaire de France.

\section{REFERENCES}

[1] P. MAJER, Two variational methods on manifolds with boundary, Topology, 34 (1995), pp. 1-12.

[2] M. Struwe, Variational Methods, Springer, Berlin, 1990. 